\title{
Hyperbolic structure on a complement of tori in the 4-sphere
}

\author{
Dubravko Ivanšić \\ Murray State University
}

\begin{abstract}
It is well known that many noncompact hyperbolic 3-manifolds are topologically complements of links in the 3 -sphere. We extend this phenomenon to dimension 4 by exhibiting an example of a noncompact hyperbolic 4-manifold that is topologically the complement of 5 tori in the 4-sphere. We also exhibit examples of hyperbolic manifolds that are complements of $5 n$ tori in a simply-connected 4-manifold with Euler characteristic $2 n$. All the examples are based on a construction of Ratcliffe and Tschantz, who produced 1171 noncompact hyperbolic manifolds with Euler characteristic 1. Our examples are finite covers of the Ratcliffe-Tschantz manifold with the biggest symmetry group.
\end{abstract}

\section{Introduction}

Let $\mathbf{H}^{n}$ be the $n$-dimensional hyperbolic space and let $G$ be a discrete subgroup of Isom $\mathbf{H}^{n}$, the isometries of $\mathbf{H}^{n}$. If $G$ is torsion-free, then $M=\mathbf{H}^{n} / G$ is a hyperbolic manifold of dimension $n$. In this paper, the term "hyperbolic manifold" will always be used for a manifold that is also complete, noncompact and has finite volume. Such a manifold $M$ is the interior of a compact manifold with boundary $\bar{M}$ (see, for example, [1]). Every boundary component of $\bar{M}$ is a compact flat (Euclidean) manifold, i.e. a manifold of the form $\mathbf{R}^{n-1} / K$, where $K$ is a discrete subgroup of Isom $\mathbf{R}^{n-1}$, the isometries of $\mathbf{R}^{n-1}$.

We say that $M$ is a (codimension-2) complement in a closed $n$-manifold $N$ if $M=N-A$, where $A$ is a closed $(n-2)$-submanifold of $N$ that has a tubular neighborhood and has as many components as $\partial \bar{M}$. Typically one would like $N$ to be a familiar manifold, such as $S^{n}$.

It is a well-known fact (see $[9,11]$ ) that many hyperbolic 3 -manifolds are complements of links in the 3 -sphere. The main purpose of this paper is to generalize this phenomenon 
to dimension 4, that is, to provide an example of a hyperbolic 4-manifold that is a complement inside the 4-sphere and to provide some examples where the hyperbolic manifolds are complements in other simply-connected 4-manifolds.

In [5] we considered the general problem of when $M$ may be thought of as a complement. Let $M=N-A$ be a complement. Then every component of $\partial \bar{M}$ must be an $S^{1}$-bundle since it will be the boundary of a tubular neighborhood of a component of $A$, and every component of $A$ must be a flat $(n-2)$-manifold, because flat manifolds are $S^{1}$-bundles only over flat manifolds (see [5]). In the 3-dimensional case, all components of $\partial \bar{M}$ are either tori or Klein bottles, both of which are $S^{1}$-bundles over $S^{1}$, so every hyperbolic 3-manifold is a complement. A slight problem arises for $n \geq 4$ where there exist $(n-1)$-dimensional flat manifolds that are not $S^{1}$-bundles. It was shown by Nimershiem in [6] that every closed, connected flat 3-manifold is a boundary component of some hyperbolic 4-manifold, so not every hyperbolic 4-manifold is a complement.

We now focus on the case $n=4$. Again, let $M=N-A$. Then components of $A$ can only be tori or Klein bottles. Furthermore, we proved in [5] that for a fixed $N$ there exist at most finitely many nonisometric hyperbolic manifolds that are complements in $N$. This is a consequence of the following three facts: that necessarily $\chi(M)=\chi(N)$, that $\operatorname{Vol}(M)=4 \pi^{2} / 3 \cdot \chi(M)$ (Gauss-Bonnet theorem for hyperbolic 4-manifolds), and that there are finitely many nonisometric hyperbolic 4-manifolds with volume less than a given number (Wang's theorem, [10]). Therefore, there exist only finitely many hyperbolic manifolds that are complements in $S^{4}$ and they must have Euler characteristic 2.

A good part for the reason that some hyperbolic 4-manifolds have not already been identified as complements is that there is a small number of examples in the literature that are given in such a way that their topology can be handled more or less easily. One way to give a hyperbolic manifold that allows for relatively easy computation of topological invariants is to represent it as a hyperbolic polyhedron whose faces have been identified via hyperbolic isometries. Such constructions have been done by Ratcliffe and Tschantz [8], Nimershiem [6] and the author [4] and we looked for complement candidates among them, primarily among examples with low Euler characteristic.

Ratcliffe and Tschantz used a computer to find different ways of pairing the sides of a 24-faced hyperbolic polyhedron that yield a hyperbolic manifold. They found 1171 nonisometric examples with Euler characteristic 1, most of them unorientable. In our search for complement examples we tested some suitable Ratcliffe-Tschantz manifolds (their boundary components had to be $S^{1}$-bundles) and found an example whose double cover is a complement in $S^{4}$. The main result of this paper is the following: 
Theorem 4.3 Let $M$ be the Ratcliffe-Tschantz manifold no. 1011. Then $M$ is a complement of 5 tori in a 4-manifold $N$ with $\pi_{1} N=\mathbf{Z}_{2}$. The orientable double cover of $M$ is the complement of 5 tori in $S^{4}$. Furthermore, the orientable double cover has cyclic covers of any index $n$ that are complements of $4 n+1$ tori in a simply-connected closed manifold $N$ with $\chi(N)=2 n$.

There are two examples by the author that have Euler characteristic $2, P / \Phi_{1}$ and $P / \Phi_{2}$, in the notation of [4]. It turns out that $P / \Phi_{2}$ has boundary components that are not $S^{1}$ bundles. We also showed that $P / \Phi_{1}$ is a complement in a 4-manifold with fundamental group $\mathbf{Z}_{2}$ and hence has a double cover that is a complement in a simply-connected 4-manifold. Nimershiem's examples are complements when all their ends are $S^{1}$-bundles, however, they have higher Euler characteristics and have not been studied by the author from the point of view of being a complement in a familiar 4-manifold.

The large number (1171) of Ratcliffe-Tschantz manifolds may give rise to other examples of complements in the 4-sphere. However, a criterion in $\S 5$ rules out most of these manifolds, leaving about 346 (hand count) of them eligible for having a double cover that is a complement in the 4-sphere. At this point, we can claim only one example with that property, the mentioned manifold no. 1011.

Acknowledgement. Practically all of the work in this paper was done while the author held a temporary position at the George Washington University. I take this opportunity to thank the GWU Department of Mathematics for providing a very congenial atmosphere and being very supportive of all my efforts during the years I was there.

\section{Hyperbolic manifolds as complements}

The following theorem summarizes some results from [5] that we will use in this paper.

Theorem 2.1 Let $E=\mathbf{R}^{n-1} / K$ be a compact flat $(n-1)$-manifold, where $K$ is a discrete subgroup of Isom $\mathbf{R}^{n-1}=\left\{A x+a \mid A \in O(n), a \in \mathbf{R}^{n-1}\right\}$. Then $E$ is an $S^{1}$-bundle over a manifold $B$ if and only if there exists a translation $t \in K$ such that $\langle t\rangle$ is a normal subgroup of $K$ and $t$ is not a power of any element of $K$ other than $t^{ \pm 1}$. If the translation is given by $x \mapsto x+v$ the normality of $\langle t\rangle$ can be expressed as $A v= \pm v$ for every $A$ such that $A x+a$ is an element of $K$. Furthermore if $n-1 \neq 4,5$ (we are interested in $n-1=3$ in this paper) the manifold $B$ above is a flat manifold.

Let $M=\mathbf{H}^{n} / G$ be a hyperbolic $n$-manifold that is a complement in some closed manifold $N$, that is $M=N-A$ as in $\S 1$. Let $\partial \bar{M}=E_{1} \cup \cdots \cup E_{m}$, where each $E_{i}$ is a flat $(n-1)$ manifold. For simplicity, we often imprecisely say the $E_{i}$ 's are boundary components of $M$ 
as well. We recall that $\pi_{1} E_{i} \stackrel{\text { inclusion }}{\longrightarrow} \pi_{1} M$ is injective. Every boundary component of $\bar{M}$ corresponds to a conjugacy class of parabolic subgroups in $G$, that is, groups consisting only of parabolic hyperbolic isometries that fix a point $v$ in $\partial \mathbf{H}^{n}$. Parabolic isometries also fix every horosphere centered at $v$. Inside the horosphere, whose inherited metric is isometric to $\mathbf{R}^{n-1}$, they act as Euclidean isometries. Each $E_{i}$ is an $S^{1}$-bundle over a component $B_{i}$ of $A$. Let $t_{i}$ denote the element of $\pi_{1} E_{i} \subset \pi_{1} M$ that represents the fiber of the $S^{1}$-bundle $E_{i}$. Then, by Theorem 2.1, each $t_{i}$ must be a translation in this group and $\left\langle t_{i}\right\rangle$ is a normal subgroup of $\pi_{1} E_{i}$. We call such an element of $\pi_{1} E_{i}$ a normal translation. Furthermore, if $t_{i}$ is not a power of any other element of $K$ other than $t_{i}^{ \pm 1}$ we call $t_{i}$ primitive in $\pi_{1} E_{i}$. We have

Proposition 2.2 Let $M$ be a complement in $N$ and let $\partial \bar{M}=E_{1} \cup \cdots \cup E_{m}$. If $t_{1}, \ldots, t_{m} \in$ $\pi_{1} M$ represent fibers of the $S^{1}$-bundles $E_{1}, \ldots, E_{m}$ then $\pi_{1} N=\pi_{1} M /\left\langle\left\langle t_{1}, \ldots, t_{m}\right\rangle\right\rangle$, where $\langle\langle A\rangle\rangle$ denotes the normal closure of a subset $A \subset \pi_{1} M$. In other words, if we have a presentation for $M$, the presentation for $N$ is obtained by adding relators $t_{1}=1, \ldots, t_{m}=1$.

Proof. We may assume $\partial \bar{M}$ has only one component $E$ - if there are more, the claim follows by induction. Let $t$ represent the $S^{1}$-fiber in $E$ and let $B$ be the base manifold in the $S^{1}$-bundle. Let $D$ be the 2 -disc bundle over $B$ obtained by filling out every $S^{1}$-fiber by a disc so that $\partial D=E$ and $N=\bar{M} \cup_{E} D$ and let $p: D \rightarrow B$ be the fiber projection. By van Kampen's theorem

$$
\pi_{1} N=\pi_{1} M * \pi_{1} D /\left\langle\left\langle i_{1 *}(e) i_{2 *}(e)^{-1}, e \in \pi_{1} E\right\rangle\right\rangle,
$$

where $i_{1}: E \rightarrow M$ and $i_{2}: E \rightarrow D$ are inclusions. However $p_{*}: \pi_{1} D \rightarrow \pi_{1} B$ is an isomorphism and we may consider $\pi_{1} E$ as a subgroup of $\pi_{1} M$ so the above group is $\pi_{1} M * \pi_{1} B /\left\langle\left\langle e p_{*}(e)^{-1}, e \in \pi_{1} E\right\rangle\right\rangle$. The inclusion map $\pi_{1} M \rightarrow \pi_{1} M * \pi_{1} B$ induces a map $\pi_{1} M /\langle\langle t\rangle\rangle \stackrel{\phi}{\rightarrow} \pi_{1} M * \pi_{1} B /\left\langle\left\langle e p_{*}(e)^{-1}, e \in \pi_{1} E\right\rangle\right\rangle$. We show that $\phi$ is an isomorphism. It is surjective, since for every element $b \in \pi_{1} B$ we have $b=p_{*}(e)$, hence $[b]=\left[e p_{*}(e)^{-1} p_{*}(e)\right]=$ $[e] \in \operatorname{im} \phi$.

Now suppose that $\phi([g])=1$ for some $g \in \pi_{1} M$. Then $g=\prod x_{i}\left(e_{i} p_{*}\left(e_{i}\right)^{-1}\right)^{ \pm 1} x_{i}^{-1}$ where $e_{i} \in \pi_{1} E$ and $x_{i} \in \pi_{1} M * \pi_{1} B$ is a product of elements in $\pi_{1} M$ and $\pi_{1} B$. However, note that a conjugate by $b \in \pi_{1} B$, where $b=p_{*}(e)$, is $b y b^{-1}=e\left(e^{-1} p_{*}(e)\right) y\left(e^{-1} p_{*}(e)\right)^{-1} e^{-1}$, so if $y$ has the form $\prod g_{j}\left(e_{j} p_{*}\left(e_{j}\right)^{-1}\right)^{ \pm 1} g_{j}^{-1}, g_{j} \in \pi_{1} M$ then

$$
\begin{gathered}
b y b^{-1}=\prod b g_{j}\left(e_{j} p_{*}\left(e_{j}\right)^{-1}\right)^{ \pm 1} g_{j}^{-1} b^{-1}=\prod e\left(e^{-1} p_{*}(e)\right) g_{j}\left(e_{j} p_{*}\left(e_{j}\right)^{-1}\right)^{ \pm 1} g_{j}^{-1}\left(e^{-1} p_{*}(e)\right)^{-1} e^{-1}= \\
\prod e\left(e^{-1} p_{*}(e)\right) e^{-1}\left(e g_{j}\right)\left(e_{j} p_{*}\left(e_{j}\right)^{-1}\right)^{ \pm 1}\left(e g_{j}\right)^{-1} e\left(e^{-1} p_{*}(e)\right)^{-1} e^{-1}
\end{gathered}
$$


which has the same form as $y$ since $e \in \pi_{1} M$. By repeatedly applying this observation we can transform the representation of $g$ to $g=\prod g_{i}\left(e_{i} p_{*}\left(e_{i}\right)^{-1}\right)^{ \pm 1} g_{i}^{-1}, g_{i} \in \pi_{1} M$. This equation is in $\pi_{1} M * \pi_{1} B$ : in order to satisfy it, all the elements of $\pi_{1} B$, namely $p_{*}\left(e_{i}\right)^{-1}$, must cancel. Then either $p_{*}\left(e_{i}\right)=1$ for all $i$ which implies that $e_{i}=t^{k_{i}}$, so $g=\prod g_{i} t^{ \pm k_{i}} g_{i}^{-1}$ in which case $[g]=1$ in $\pi_{1} M /\langle\langle t\rangle\rangle$, or there exists an $i$ so that the term between $p_{*}\left(e_{i}\right)^{ \pm 1}$ and $p_{*}\left(e_{i+1}\right)^{ \pm 1}$ cancels. It is not hard to see (there are four cases depending on the exponents \pm 1 ) that the product then reduces to a product of the same form with one fewer term, so the argument is finished by induction.

In order to simplify computations showing that a proposed $M$ is a complement, we need the following propositions.

Proposition 2.3 Let $\tilde{X} \stackrel{p}{\rightarrow} X$ be the covering space of a manifold $X$ corresponding to a normal subgroup $H$ of $G=\pi_{1} X$. If $E$ is a path-connected subset of $X$ (e.g. a submanifold), and $K=i_{*}\left(\pi_{1}(E)\right)$, where $E \stackrel{i}{\rightarrow} X$ is the inclusion, then the number of path-components of $p^{-1}(E)$ is equal to the index of $K H$ in $G$. Note that this is the same as the index in $G / H$ of the image of $K$ under the quotient map $G \rightarrow G / H$.

Proof. Choose $x_{0} \in E$ and let $Y=p^{-1}\left(x_{0}\right)$. If we choose $y_{0} \in Y$ there is a standard correspondence $G / H \leftrightarrow Y$ given by $g H \leftrightarrow$ (the endpoint of the lift of $g$ starting at $y_{0}$ ) that we can compose to a map $G / H \stackrel{\phi}{\rightarrow} Y / \sim$, where $y_{1} \sim y_{2}$ if both are in the same path-component of $p^{-1}(E)$. Suppose $\phi\left(g_{1} H\right)=\phi\left(g_{2} H\right)$. Then loops $g_{1}$ and $g_{2}$ lift to paths starting with $y_{0}$ and ending with $y_{1}$ and $y_{2}$ respectively and there is a path $\tilde{k}$ from $y_{1}$ to $y_{2}$ in $p^{-1}(E)$. Taking $k=p \circ \tilde{k}$ gives a loop in $E$ whose homotopy class is in $K$ and the lift of $g_{1} k$ has the same endpoint as $g_{2}$, thus $g_{1} k H=g_{2} H$, thus $g_{2}^{-1} g_{1}=h k^{-1}$ for some $h \in H$. Now $h k^{-1}=k^{-1} k h k^{-1} h$, so $g_{2}^{-1} g_{1}=k^{-1} h_{1}$, for some $h_{1} \in H$ because $H$ is normal. Hence $g_{1}$ and $g_{2}$ are in the same class of $K H$. (Note that, due to normality of $H$, we can write any element of $K H$ as $k h$ for some $k \in K$ and $h \in H$.) Conversely, if $g_{1} K H=g_{2} K H$ then $g_{2}=g_{1} k h$ for some $k \in K, h \in H$. Then the lifts of $g_{1} k$ and $g_{2}$ starting at $y_{0}$ have the same endpoint $y_{2}$, but since we may assume that the loop $k$ lies in $E$ then the endpoint $y_{1}$ of $g_{1}$ is connected to $y_{2}$ by a lift of $k$ starting at $y_{1}$, but this path is in $p^{-1}(E)$, so $\phi\left(g_{1} H\right)=\phi\left(g_{2} H\right)$. Therefore, $\phi$ induces a bijection between the set of path components of $p^{-1}(E)$ (same as $Y / \sim$ ) and the set of left cosets of $K H$.

Note also that the number of preimages of $x_{0}$ that are in the same path-component of $p^{-1}(E)$ is equal to the index of $H$ in $K H$, which, by the standard isomorphism theorem $K H / H \cong K /(K \cap H)$ is equal to the index of $K \cap H$ in $K$. 
Proposition 2.4 Let $M$ be a hyperbolic manifold with $\partial \bar{M}=E_{1} \cup \cdots \cup E_{m}$ and let $t_{1} \in$ $\pi_{1} E_{1}, \ldots, t_{m} \in \pi_{1} E_{m}$ be normal translations. Suppose that $\pi_{1} M /\left\langle\left\langle t_{1}, \ldots, t_{m}\right\rangle\right\rangle$ is a finite group of order $l$ and that $t_{i}$ is primitive in $\pi_{1} E_{i} \cap\left\langle\left\langle t_{1}, \ldots, t_{m}\right\rangle\right\rangle$ for every $i=1, \ldots, m$.

Then $M$ has an l-fold normal cover that is a complement inside a simply-connected closed manifold $N$.

Proof. Set $G=\pi_{1} M, H=\left\langle\left\langle t_{1}, \ldots, t_{m}\right\rangle\right\rangle, K_{i}=\pi_{1} E_{i}, i=1, \ldots, m$ and let $t_{1}, \ldots, t_{m}$ be normal translations satisfying conditions of the theorem and let $\Phi=G / H$, where $q: G \rightarrow \Phi$ is the quotient map. For every $i$, we may choose a finite set $R_{i} \subset K_{i}$ that is a transversal (i.e. a set of coset representatives) of $K_{i} \cap H$ in $K_{i}$ and a finite set $S_{i} \subset G$ that is a transversal of $K_{i} H$ in $G$. Any element $g \in G$ may be written as $r_{i} s_{i} h$ for some $r_{i} \in R_{i}, s_{i} \in S_{i}, h \in H$. The conjugate $g^{-1} t_{i} g$ can then be written as $h^{-1} s_{i}^{-1} r_{i}^{-1} t_{i} r_{i} s_{i} h=h^{-1} s_{i}^{-1} t_{i}^{ \pm 1} s_{i} h$ since $t_{i}$ is a normal translation. The elements of $\left\langle\left\langle t_{1}, \ldots, t_{m}\right\rangle\right\rangle$ are products of form $\prod g_{j}^{-1} t_{i_{j}}^{l_{j}} g_{j}$ which can be written as $\prod h_{j}^{-1} s_{j}^{-1} r_{j}^{-1} t_{i_{j}}^{l_{j}} r_{j} s_{j} h_{j}=\prod h_{j}^{-1} s_{j}^{-1} t_{i_{j}}^{ \pm l_{j}} s_{j} h_{j}$ where $h_{j} \in H, s_{j} \in S_{i_{j}}$ and $r_{j} \in R_{i_{j}}$. Therefore, if $\langle\langle A\rangle\rangle_{H}$ denotes the normal closure in $H$ of a subset $A$ of $H$, then $H=\left\langle\left\langle t_{1}, \ldots, t_{m}\right\rangle\right\rangle=\left\langle\left\langle s_{i}^{-1} t_{i} s_{i} \mid s_{i} \in S_{i}, i=1, \ldots, m\right\rangle\right\rangle_{H}$.

By Proposition 2.3, $p^{-1}\left(E_{i}\right)$ has $\left[\Phi: q\left(K_{i}\right)\right]=\left|S_{i}\right|$ components, $i=1, \ldots, m$. Restricting $p$ to each of the components we get a covering of $E_{i}$ corresponding to the subgroup $K_{i} \cap H \subset K_{i}$. Normality of translations $t_{1}, \ldots, t_{m}$ and the fact that each of them is primitive in $K_{i} \cap H$ ensure that each component of $p^{-1}\left(E_{i}\right)$ is a fiber bundle and that we may choose as a fiber any lift of a loop representing the element $t_{i}$ that is contained in $p^{-1}\left(E_{i}\right)$. Therefore, the cover of $M$ corresponding to $H \subset \pi_{1} M$ is going to be a complement in some closed manifold $N$. What is $\pi_{1} N$ ? As seen above, components of $p^{-1}\left(E_{i}\right)$ are in 1-1 correspondence with elements of $S_{i}$ and a fiber in each component represents the element $s_{i}^{-1} t_{i} s_{i}$, where $s_{i}$ ranges through $S_{i}$. Inserting 2-disc-bundles that close off components of $p^{-1}\left(E_{i}\right)$ gives us $N$, whose fundamental group, according to Proposition 2.2, is $H /\left\langle\left\langle s_{i}^{-1} t_{i} s_{i} \mid s_{i} \in S_{i}, i=1, \ldots m\right\rangle\right\rangle_{H}=H / H=1$.

\section{Moving around a tiling of $\mathbf{H}^{n}$}

Let $G$ be a discrete subgroup of Isom $\mathbf{H}^{n}$ that is generated by side-pairings of its finite-sided fundamental polyhedron $P$ (see [7] or [4]). If $S$ is a side of $P$, let $s$ be the side-pairing that sends $S$ to its pair $S^{\prime}$ (thus, $s S=S^{\prime}$ ). Then $s^{-1}$ will pair $S^{\prime}$ to $S$. For every point $x$ in $P$ let $[x]$, the cycle of $x$, be the set of all points in $P$ that are obtained by sending $x$ around $P$ via side-pairing transformations. As in [7], for every $x \in P$ let $\omega(x)$ be the measure of the "spatial angle" that $P$ subtends at $x$, that is $\omega(x)=\operatorname{Vol}(B(x, r) \cap P) / \operatorname{Vol} B(x, r)$, where $B(x, r)$ is a ball around $x$ with radius $r$ small enough so that $B(x, r)$ intersects only the sides 
on which $x$ lies. Set $\omega[x]=\sum_{y \in[x]} \omega(y)$. Then $G$ will be a torsion free group if and only if $\omega[x]=1$ for every $x \in P$ (see [7]). Note that for a polyheron $P$ with ideal vertices $[x]$ is well-defined for an ideal vertex as well.

Since $P$ is a fundamental polyhedron, $\mathbf{H}^{n}=\cup_{g \in G} g P$ where $g_{1}(\operatorname{int} P) \cap g_{2}($ int $P)=\emptyset$ for $g_{1} \neq g_{2}$ and any two polyhedra $g_{1} P$ and $g_{2} P$ either do not intersect or intersect along one of their $k$-faces. Imagine a piecewise-linear path whose every vertex is in int $g P$ for some $g \in G$ and whose edges join vertices in adjacent translates of $P$ so that the edge between two consecutive vertices passes through the interiors of the sides that the adjacent translates share. If the translates are $g_{1} P$ and $g_{2} P$ and we exited through side $g_{1} S$ of the polyhedron $g_{1} P$, then we entered $g_{2} P$ through side $g_{2} S^{\prime}$, where $S^{\prime}$ is the side paired to $S$ under the sidepairing of $P$. If $s$ is the transformation that pairs $S$ to $S^{\prime}$, then $g_{2}=g_{1} s^{-1}$. Thus, if we start in translate $g P$ and the path exits, sequentially, through the translates of sides $S_{1}, \ldots, S_{m}$ of $P$, then the vertices of the path are in translates $g P, g s_{1}^{-1} P, g s_{1}^{-1} s_{2}^{-1} P, \ldots, g s_{1}^{-1} \ldots s_{m}^{-1} P$.

Let $H$ be a finite-index subgroup of $G$ with transversal $X$, so $G=\cup_{x \in X} H x$. Then $Q=\cup_{x \in X} x P$ is a fundamental polyhedron for $H$ (elements of $X$ can be chosen so that $Q$ is connected). How are sides of $Q$ paired in order to generate $H$ ? If $R$ is a side of $Q$, then $R=x S$ for some side $S$ of $P$. Let $f$ be the transformation that pairs $R$ to a side $R^{\prime}$ of $Q$. Adjacent to $Q$ on the other side of $R^{\prime}$ is $f Q$ and $R^{\prime}=y S^{\prime}$ for some $y \in X$. Now in the tiling by $P$, translates $f x P$ and $y P$ meet along paired sides of $P$ and one gets from $y P$ to $f x P$ by exiting through $y S^{\prime}$. By the above we get $f x=y s$, which implies $y s x^{-1}=f \in H$ making $y$ the coset representative for $H x s^{-1}$. Therefore, side $x S$ gets paired to side $y S^{\prime}$ via $f=y s x^{-1}$, where $S^{\prime}$ is the pair of $S$ under side-pairing $s$ of $P$ and $y$ is the element of the transversal of $H$ that represents the coset $H x s^{-1}$.

We now turn to the special case where $G$ is generated by reflections in the sides of a polyhedron $P$. The following proposition is inspired by and generalizes a claim from [8] that was specific to the polyhedra $P$ and $Q$ Ratcliffe and Tschantz were considering.

Proposition 3.1 Let $G$ be generated by reflections in the sides of a polyhedron $P$ and let $H$ be a finite-index subgroup of $G$ so that its transversal is a finite group $K$. (In other words, $G=H K$ and $H \cap K=1$.) Then the fundamental polyhedron for $H$ is $Q=\cup_{k \in K} k P$ which is invariant under $K$. Furthermore, any side $R$ of $Q$ is paired to a side $R^{\prime}$ of $Q$ by a transformation of the form $k r$, where $k \in K$ is such that $k(R)=R^{\prime}$ and $r$ is a reflection in $R$. Alternatively, $R$ is paired to $R^{\prime}$ by a transformation of the form $r^{\prime} k$, where $r^{\prime}$ is a reflection in $R^{\prime}$ and $k \in K$ such that $k(R)=R^{\prime}$.

Proof. Applying the above discussion to this situation, we see that side $R=k_{1} S$ is paired to side $R^{\prime}=k_{2} S^{\prime}$ via $f=k_{2} s k_{1}^{-1}$, where $s$ is a reflection in $S$. Then $f=\left(k_{2} k_{1}^{-1}\right)\left(k_{1} s k_{1}^{-1}\right)$, but $r=k_{1} s k_{1}^{-1}$ is a reflection in $k_{1} S=R$ and $k=k_{2} k_{1}^{-1}$ is an element of $K$ that sends 
$R=k_{1} S$ to $R^{\prime}=k_{2} S$, so $f=k r$. Now we note that $k r=k r k^{-1} k$ and $r^{\prime}=k r k^{-1}$ is the reflection in $k R=R^{\prime}$.

In this paper we will be interested in finding generators of parabolic subroups of $G$ that correspond to fundamental groups of boundary components of the manifold. Let $v$ be an ideal vertex of $P$ and let $X_{v}$ be the finite set of transformations in $G$ that send vertices in the cycle $[v]$ to $v$. Thus for $w \in[v]$ there is a unique $x \in X_{v}$ so that $x w=v$. The subgroup $G_{v}=\operatorname{stab}_{v} G=\{g \in G \mid g v=v\}$ is the fundamental group of the boundary component of $M=\mathbf{H}^{n} / G$ that corresponds to the ideal vertex $v$. Since the manifold is complete, $G_{v}$ consists only of parabolic elements, so it will fix every horosphere centered at $v$. Choose a sufficiently small horosphere $C$ centered at $v$ that intersects only those sides of $P$ in whose closure $v$ is. Then $G_{v}$ is a group of Euclidean isometries of $C \cong \mathbf{R}^{n-1}$. At other vertices in $[v]$ we choose horospheres $x^{-1} C$, where $x \in X_{v}$. The set $\left(x^{-1} C\right) \cap P$ is a Euclidean polyhedron in the horosphere $x^{-1} C$ whose sides are intersections of sides of $P$ with the horosphere $x^{-1} C$.

The fundamental polyhedron for $G_{v}$ and its side-pairing transformations are found in much the same way as the fundamental polyhedron of a finite-index subgroup of $G$, above. First of all, the fundamental polyhedron for $G_{v}$ is $P_{v}=\left(\cup_{x \in X_{v}} x P\right) \cap C$ where we can choose elements of $X_{v}$ so that the above union is connected. Let $x_{1} S$ be a side of $x_{1} P \cap C, v_{1} \in[v]$ so $x_{1} v_{1}=v$; let $s$ be the side-pairing transformation that sends $S$ to $S^{\prime}$, and let $v_{2}=s v_{1}$. (Note that $v_{1}$ is on side $S$ and $v_{2}$ is on side $S^{\prime}$.) Then $x_{1} S \cap C$ will be paired to $x_{2} S^{\prime} \cap C$ and the side-pairing transformation is $x_{2} s x_{1}^{-1}$ - note that this transformation is in $G_{v}$.

We will make use of the following proposition. For simplicity of notation, when we discuss the fundamental polyhedron of $G_{v}$, we are assuming the intersection of the polyhedron $P$ and its sides with the horosphere $C$ so we drop the " $\cap C$ ".

Proposition 3.2 Let $G, P, K, H$ and $Q$ be as in Proposition 3.1, $v$ an ideal vertex of $P$, $G_{v}=\operatorname{stab}_{v} G$, and let $X_{v}$ be as above. Then $Q_{v}=\cup_{x \in X_{v}} x Q$ is the fundamental polyhedron for $G_{v}$. Let side $x S$ of $Q_{v}$ be paired to $y S^{\prime}$ via $f=y s x^{-1}$ where $x, y \in X_{v}$. Consider a piecewise linear path (as above) that starts in $x Q$, goes to $y Q$ and exits through side $y S^{\prime}$ of $y Q$. Suppose the path from $x Q$ to $y Q$ passes through different translates $x_{1} Q=x Q, x_{2} Q, \ldots, x_{m} Q, y Q$ of $Q$, exiting every time through side $x_{i} S_{i}$ of $x_{i} Q$ and finally exiting through $y S^{\prime}$. If $x=y$ set $m=0$. This means that $y=x s_{1}^{-1} s_{2}^{-1} \ldots s_{m}^{-1}$, where $s_{i}$ is the transformation that pairs $S_{i}$.

Then the side-pairing $f$ can be written as $f=\left(q q_{m} \ldots q_{1}\right) x\left(k_{1}^{-1} \ldots k_{m}^{-1} k^{-1}\right) x^{-1}$ where $q_{i}$ is the reflection in the plane $x_{i} S_{i}, q$ is the reflection in plane $y S^{\prime}$, and $k_{i}$ and $k$ are elements of $K$ in the decompositions of side pairings $s_{i}$ and $s^{-1}$, respectively, into form $k r$ as in Proposition 3.1.

In other words, as we go from $x P$ to ysP we pass through a sequence of hyperplanes containing $v$. The side-pairing that sends $x S$ to $y S^{\prime}$ is the product of reflections in those 
hyperplanes and the conjugate of products of elements of $K$ that correspond to the sidepairings of sides whose translates we exit through.

Proof. Let $x=1$. We first prove the claim $s_{1}^{-1} \ldots s_{m}^{-1}=\left(q_{m} \ldots q_{1}\right)\left(k_{1}^{-1} \ldots k_{m}^{-1}\right)$ by induction on $m$. Note that $x_{2}=s_{1}^{-1}, x_{3}=s_{1}^{-1} s_{2}^{-1}$ and so on. For $m=1$ we have $s_{1}^{-1}=\left(k_{1} r_{1}\right)^{-1}=$ $r_{1} k_{1}^{-1}$, and $r_{1}$ is a reflection in $x_{1} S_{1}=S_{1}$. Suppose the claim holds for $m-1$. Then $s_{1}^{-1} \ldots s_{m-1}^{-1} s_{m}^{-1}=\left(s_{1}^{-1} \ldots s_{m-1}^{-1}\right) r_{m}^{-1}\left(s_{m-1} \ldots s_{1}\right)\left(s_{1}^{-1} \ldots s_{m-1}^{-1}\right) k_{m}^{-1}=q_{m}\left(s_{1}^{-1} \ldots s_{m-1}^{-1}\right) k_{m}^{-1}=$ $q_{m}\left(q_{m-1} \ldots q_{1} k_{1}^{-1} \ldots k_{m-1}^{-1}\right) k_{m}^{-1}$. Here we used that $\left(s_{1}^{-1} \ldots s_{m-1}^{-1}\right) r_{m}^{-1}\left(s_{m-1} \ldots s_{1}\right)$ is a reflection in the plane $s_{1}^{-1} \ldots s_{m-1}^{-1} S_{m}=x_{m} S_{m}$. Of course now $s_{1}^{-1} \ldots s_{m}^{-1} s=s_{1}^{-1} \ldots s_{m}^{-1}\left(s^{-1}\right)^{-1}$ and the claim follows from what we just proved since $s^{-1}=k r$.

For a general $x$ notice that the element of $G$ sending $x$ to $y s=x s_{1}^{-1} \ldots s_{m}^{-1} s$ is given by $x s_{1}^{-1} \ldots x_{m}^{-1} s x^{-1}=\left(x q q_{m} \ldots q_{1} x^{-1}\right)\left(x k_{1}^{-1} \ldots k_{m}^{-1} k^{-1} x^{-1}\right)$. However, if $q_{i}$ is a reflection in $s_{1}^{-1} \ldots s_{i-1}^{-1} S_{i}$ then $x q_{i} x^{-1}$ is a reflection in $x s_{1}^{-1} \ldots s_{i-1}^{-1} S_{i}=x_{i} S_{i}$ and the claim is proved.

Remark 3.3 Note that $k_{1}^{-1} \ldots k_{m}^{-1} k^{-1}$ is an element of $K$ that preserves $Q$, the vertex $x^{-1} v$ and $x^{-1} C$, hence $x\left(k_{1}^{-1} \ldots k_{m}^{-1} k^{-1}\right) x^{-1}$ preserves $v, C$ and $x Q$, so the side-pairing is a transformation that keeps $x Q$ fixed followed by reflections in the above hyperplanes which contain $v$.

\section{Example of a complement in the 4-sphere}

We now recall Ratcliffe and Tschantz's 24-sided polyhedron (see [8]) and a side-pairing that yields an unorientable manifold with Euler characteristic 1.

We use the ball model of hyperbolic 4-space $\mathbf{H}^{4}$, so $\mathbf{H}^{4}=B^{4}$, the unit 4-ball, with the differential metric $d s^{2}=\frac{4|d x|^{2}}{\left(1-|x|^{2}\right)^{2}}$. Hyperplanes in $\mathbf{H}^{4}$ are round 3 -spheres in $\mathbf{R}^{4}$ that are perpendicular to $\partial B^{4}$.

Consider the 24 spheres of radius 1 that are centered at points whose two coordinates are \pm 1 and the other two are 0 . Each of those spheres is perpendicular to $\partial \mathbf{H}^{4}$ and any two of them either intersect at a right angle, are tangential with tangent point on $\partial \mathbf{H}^{4}$ or are disjoint. The spheres thus determine 24 hyperplanes that intersect at right angles. Let $Q$ be the polyhedron that is the intersection of the 24 half-spaces determined by the hyperplanes, where we always take the half-space that contains the origin. We will denote the sides of $Q$ and the hyperplanes that they lie on by $S_{* * * *}$, where "****" is a string with two zeroes and two +'s or -'s that determines the center of the sphere on which the hyperplane lies. For example, $S_{+0-0}$ is the side lying on the sphere centered at $(1,0,-1,0)$.

The polyhedron $Q$ has ideal vertices $v_{* 000}=( \pm 1,0,0,0), v_{0 * 00}=(0, \pm 1,0,0), v_{00 * 0}=$ $(0,0, \pm 1,0), v_{000 *}=(0,0,0, \pm 1)$ and $v_{* * * *}=( \pm 1 / 2, \pm 1 / 2, \pm 1 / 2, \pm 1 / 2)$, where the "*" in the subscript is always "+" or "-". The polyhedron has no real vertices. 
Note that two sides intersect when the strings describing them have equal nonzero entries in one position and the positions of the remaining nonzero entries are different. Two sides touch at $\partial \mathbf{H}^{4}$ when they have equal nonzero entries in one position and they have opposite nonzero entries in another position or if the positions where they have nonzero entries are complementary. Furthermore, an ideal vertex will be on a side if its euclidean distance from the center of the sphere defining the side is 1 . (For example, $S_{0-+0}$ and $S_{+-00}$ intersect, $S_{0-+0}$ and $S_{0+0+}$ are disjoint, $S_{0-+0}$ and $S_{0--0}$ touch at $v_{0-00}$, and $S_{0-+0}$ and $S_{-00-}$ touch at $\left.v_{--+-}\right)$.

The intersection of the horosphere centered at an ideal vertex $v$ with the hyperplanes that contain $v$ looks like a cube because this horosphere intersects six sides that intersect either at right angles or meet only at $v$, hence their intersection with the horosphere are pairs of parallel planes that intersect at right angles. Due to symmetry of $Q$, the intersection of $Q$ with $C$ will be a cube rather than a general rectangular box.

Let $P$ denote the intersection of $Q$ with the positive hexadecant and let $K$ be the order-16 group generated by reflections in hyperplanes $x_{i}=0, i=1, \ldots, 4$. Elements of $K$ we denote as $k_{* * * *}$ where every "*" is a "+" or a "-". It is clear that $Q=K P=\{k P \mid k \in K\}$.

Ratcliffe and Tschantz investigated $\Gamma_{2}^{4} \subset$ Isom $\mathbf{H}^{n}$, the congruence two group of integral Lorentzian $5 \times 5$ matrices (see [8] for definition) and found that it is isomorphic to the group generated by reflections in the sides of the polyhedron $P$. Thus, $\Gamma_{2}^{4}=$ $\left\langle k_{-+++}, k_{+-++}, k_{++-+}, k_{+++-}, r_{++00}, r_{+0+0}, r_{0++0}, r_{+00+}, r_{0+0+}, r_{00++}\right\rangle$, where $r_{* * * *}$ is a reflection in the side $S_{* * * *}$. If $G$ is a finite-index torsion-free subgroup of $\Gamma_{2}^{4}$, then $K$ acts on the set $\Gamma_{2}^{4} / G$ freely so $K$ must divide $\left[\Gamma_{2}^{4}: G\right]$. If $G$ has minimal index 16 , then $K$ is a set of coset representatives so $Q$ will be a fundamental polyhedron for $G$, and $G$ will be generated by side-pairings of $Q$. Ratcliffe and Tschantz showed that any side-pairing transformation of $Q$ that takes side $R$ to side $R^{\prime}$ must be of form $k r$ or $r^{\prime} k$, where $k \in K$ takes $R$ to $R^{\prime}$ and $r$ and $r^{\prime}$ are reflections in $R$ and $R^{\prime}$, respectively. (This claim follows from Proposition 3.1.) Thus, a side-pairing transformation will be specified by giving an element of $K$.

Ratcliffe and Tschantz used a computer to find all the side-pairings of $Q$ for which $\omega[x]=1$ for every $x \in \partial Q$. They are the side-pairings that yield a hyperbolic manifold. The list of 1171 nonisometric manifolds that they obtained, along with their side-pairings is in their paper [8]. They also found that all sides of $Q$ labeled by a string with the two zeroes in the same place have the same corresponding $k$.

A specialization of Proposition 3.2 will be useful: 
Proposition 4.1 Let $G, Q$, and $K$ be as in proposition 3.2, specialized to any of the Ratcliffe-Tschantz examples. In this case $K=\mathbf{Z}_{2}^{4}$, in particular, it is abelian. Then, with the set-up from Proposition 3.2, a side-pairing of $Q_{v}$ can be written in form $f=\left(q q_{m} \ldots q_{1}\right) x\left(k_{1} \ldots k_{m}\right) x^{-1}=\left(q q_{m} \ldots q_{1}\right)\left(p_{j} \ldots p_{1}\right)\left(k_{1} \ldots k_{m} k\right)\left(p_{j} \ldots p_{1}\right)^{-1}$ where $p_{1}, \ldots, p_{j}$ are reflections in the hyperplanes that one passes through by going from $Q$ to $x Q$.

Proof. We only need to rewrite $x\left(k_{1}^{-1} \ldots k_{m}^{-1} k^{-1}\right) x^{-1}$. From Proposition 3.2 we can write $x=\left(p_{j} \ldots p_{1}\right)\left(h_{1}^{-1} \ldots h_{j}^{-1}\right)$ by following a path from $Q$ to $x Q$, where $h_{1}, \ldots, h_{j}$ are appropriate elements of $K$. Commutativity of $K$ now reduces $x\left(k_{1}^{-1} \ldots k_{m}^{-1} k^{-1}\right) x^{-1}$ to $\left(p_{j} \ldots p_{1}\right)\left(k_{1}^{-1} \ldots k_{m}^{-1} k^{-1}\right)\left(p_{j} \ldots p_{1}\right)^{-1}$ and the fact that every element of $K$ has order 2 allows us to write $k_{i}$ instead of $k_{i}^{-1}$.

Remark 4.2 Note that $k_{1} \ldots k_{m} k$ preserves $v$ since it preserves $w=h_{1}^{-1} \ldots h_{j}^{-1} v$ and the two transformations commute. Each $p_{i}$ is a reflection in a hyperplane containing $v$ which will make $\left(p_{j} \ldots p_{1}\right)\left(k_{1} \ldots k_{m} k\right)\left(p_{j} \ldots p_{1}\right)^{-1}$ easy to visualize: it is nothing but the transformation $k_{1} \ldots k_{m} k$ moved to $\left(p_{j} \ldots p_{1}\right) Q$.

We now show that the orientable double cover of manifold no. 1011 (call it $M$ ) in Ratcliffe and Tschantz's list is a complement in the 4-sphere and that it has covers of every even index that are complements in some other simply-connected 4-manifold. Of the 1171 Ratcliffe and Tschantz's examples, we chose to try this one because it has the largest symmetry group, which slightly simplifies the calculations.

First of all, the side-pairing for $M$ is given by transformations that we name $a, b, \ldots, k, l$ as follows ( $\mathrm{r}$ is always the reflection in the originating side):

$$
\begin{array}{lll}
S_{++00} \stackrel{a}{\rightarrow} S_{-+00} & S_{+-00} \stackrel{b}{\rightarrow} S_{--00} & a, b=k_{-+++} r \\
S_{+0+0} \stackrel{c}{\rightarrow} S_{+0-0} & S_{-0+0} \stackrel{d}{\rightarrow} S_{-0-0} & c, d=k_{++-+} r \\
S_{0++0} \stackrel{e}{\rightarrow} S_{0--0} & S_{0+-0} \stackrel{f}{\rightarrow} S_{0-+0} & e, f=k_{----} r \\
S_{+00+} \stackrel{g}{\rightarrow} S_{-00-} & S_{+00-} \stackrel{h}{\rightarrow} S_{-00+} & g, h=k_{---} r \\
S_{0+0+} \stackrel{i}{\rightarrow} S_{0-0+} & S_{0+0-} \stackrel{j}{\rightarrow} S_{0-0-} & i, j=k_{+-++} r \\
S_{00++} \stackrel{k}{\rightarrow} S_{00+-} & S_{00-+} \stackrel{b}{\rightarrow} S_{00--} & k, l=k_{+++-} r
\end{array}
$$

The presentation for the group $G$ generated by these side-pairings is obtained by performing an edge-chase (see e.g. [4]). Since there are no real vertices, every 2-face of $Q$ (an "edge", or a codimension-2 face) has an ideal vertex. We will use fundamental polyhedra $Q_{v}$, as above, to find generators and normal translations of $\pi_{1} E_{i}$, where $\partial \bar{M}=E_{1} \cup \cdots \cup E_{5}$. These polyhedra will also help with the edge chase, since each of their codimension- 2 faces 
is the intersection of a codimension-2 face of $Q$ with a horosphere $C$ and can be used to represent an edge in the cycle. All the edges in a cycle have a vertex $v$ that is an element of the cycle of some vertex, so all the edges of a cycle will be represented by a codimension- 2 face of some polyhedron $Q_{v}$. Furthermore, since every 2-face of $Q$ has an ideal vertex that is among $v_{* 000}, v_{0 * 00}, v_{00 * 0}$ and $v_{000 *}$, the codimension- 2 faces of polyhedra $Q_{v_{+000}}, Q_{v_{0+00}}$, $Q_{v_{00+0}}$ and $Q_{v_{000+}}$ will represent all the edges from all cycles.

Figure 1 contains pictures of $Q_{v_{+000}}, Q_{v_{0+00}}, Q_{v_{00+0}}, Q_{v_{000+}}$. Using Proposition 4.1 we can determine the side-pairings of each $Q_{v}$ and the generating translations of the translation subgroup of $G_{v}$ as well as which ones among them are normal.

We explain the method on the example $Q_{v_{+000}}$. The only other vertex in the cycle of the vertex $v_{+000}$ is $v_{-000}=a v_{+000}$. Hence $Q_{v_{+000}}=\left(Q \cup a^{-1} Q\right) \cap C$ will be a union of two side-by-side cubes, it is the top rectangular box depicted in Fig. 1. The strings labeling the sides of $Q_{v_{+000}}$ come from the labeling of sides of $Q$ (sides on the right cube are actually translates of the sides of $Q$ with the indicated label). The symbol $v_{-000}$ above the right cube denotes that the right cube is a translate (by $a^{-1}$ ) of $Q$ intersected with a horosphere centered at $v_{-000}$.

From Fig. 1 we can read off the cycles of edges. For example, look at $S_{+0-0} \cap S_{+00+}$. We see that

$$
S_{+00+} \cap S_{+0+0} \stackrel{c}{\rightarrow} S_{+0-0} \cap S_{+00+} \stackrel{g}{\rightarrow} S_{-00-} \cap S_{-0+0} \stackrel{d}{\rightarrow} S_{-0-0} \cap S_{-00-} \stackrel{g^{-1}}{\longrightarrow} S_{+00+} \cap S_{+0+0}
$$

and this edge cycle contributes the relation $g^{-1} d g c=1$ to the presentation of $G$.

Now for the side-pairing transformations of $Q_{v_{+000}}$. Note that under the natural identification $C \cong \mathbf{R}^{3}$ the origin of $\mathbf{R}^{n-1}$ may be taken to be the center of the cube $Q \cap C$. In Fig. 1 we indicate the directions of the coordinate axes at left - they apply to only the left cubes in the diagram, as the orientation of the right cubes is governed by the side-pairing.

Side $S_{+0+0}$ is paired to side $S_{+0-0}$ via $c$. Since $c v_{+000}=v_{+000}$ we see that $c \in G_{v_{+000}}$. By Proposition $4.1 c$ is the composite of the reflection in $S_{+0-0}$ and $k_{++-+}^{-1}$, which is a reflection in the plane $x_{3}=0$, making $c$ a translation in the $x_{3}$-direction (but with opposite orientation). We use similar reasoning to see that $a^{-1} S_{-0+0}$ is paired to $a^{-1} S_{-0-0}$ via a translation in the $x_{3}$-direction.

Side $S_{+-00}$ is paired to side $S_{--00}$ via $b$. Since $b v_{+000}=v_{-000}$ then $S_{+-00}$ will be sent to $a^{-1} S_{--00}$ by an element of $G_{v_{+000}}$. This transformation will be the composite of reflections in $S_{++00}$ and $a^{-1} S_{--00}$ (respectively, the center dividing plane and the right side of the top box in Fig. 1, respectively) and of $k_{-+++} k_{-+++}=1$, so will thus be a translation in the $x_{2}$ direction.

Side $S_{+00+}$ is paired to side $S_{-00-}$ via $g$. Since $g v_{+000}=v_{-000}$ there is an element of $G_{v_{+000}}$ that sends $S_{+00+}$ to $a^{-1} S_{-00-}$. This transformation is a composite of reflections in $S_{++00}$ 

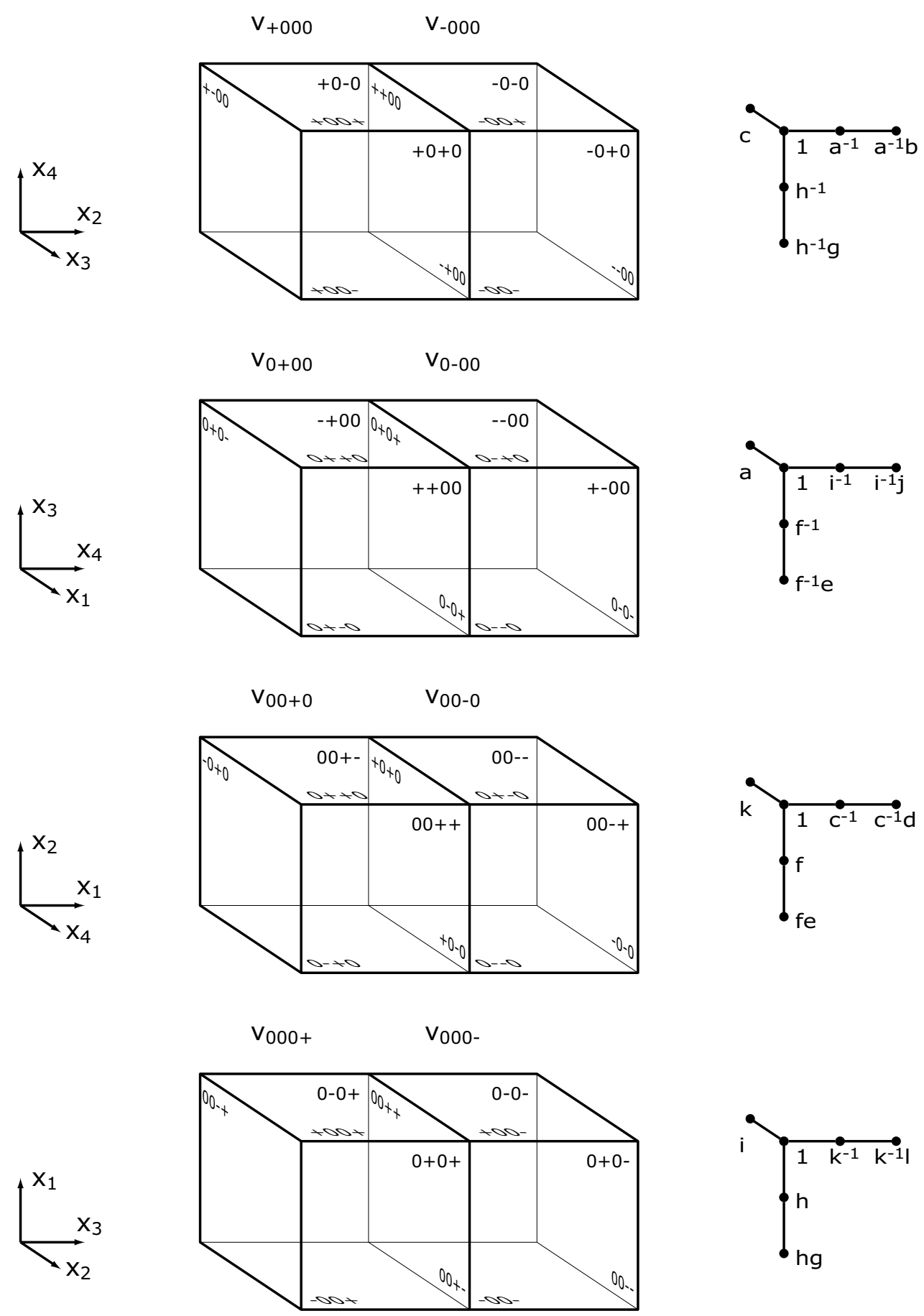

Figure 1: Fundamental polyhedra for $G_{v_{+000}}, G_{v_{0+00}}, G_{v_{00+0}}, G_{v_{000+}}$ and generators of their translation subgroups. 


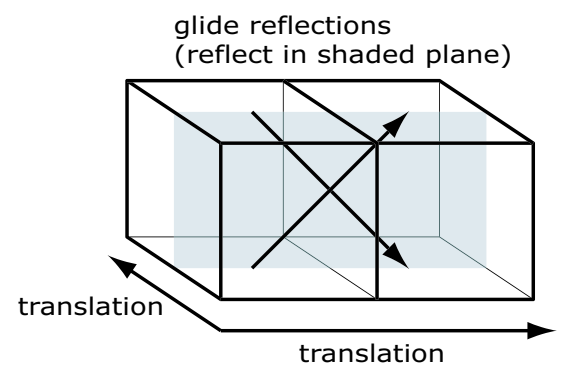

Figure 2: Side-pairing pattern for fundamental polyhedra $Q_{v_{+000}}, Q_{v_{0+00}}, Q_{v_{00+0}}, Q_{v_{000+}}$

and $a^{-1} S_{-00-}$ and $k_{-+++} k_{----}=k_{+---}$. Hence, its rotational part will be the composite of reflections in $x_{2}=0, x_{4}=0$, which are parallel to $S_{++00}$ and $a^{-1} S_{-00-}$, respectively, and $\left.k_{+---}\right|_{C}$. The composite is thus a reflection in $x_{3}=0$. It is now clear that $S_{++00}$ is sent to $a^{-1} S_{-00-}$ by a glide reflection, that is, a translation composed with a reflection in a plane containing the translation. The plane is $x_{3}=0$, the plane going through the middle of the two cubes and parallel to the front side. We use similar reasoning to see that $S_{+00-}$ is paired to $a^{-1} S_{-00+}$ via a glide reflection, where the reflecting is again performed in the plane $x_{3}=0$.

The pattern of side-pairings, which is the same for each of the rectangular boxes $Q_{v_{+000}}$, $Q_{v_{0+00}}, Q_{v_{00+0}}$ and $Q_{v_{000+}}$ is illustrated on Fig. 2 - arrows indicate translations and it is noted if a translation is composed with any reflections. We can see that $G_{v_{+000}}$ will be generated by three elements: the translation $c$ that pairs the front and back sides, the translation $a^{-1} b$ that pairs the left and right sides and the glide reflection $a^{-1} g$ that pairs half of the top side with half of the bottom side. (The second glide reflection is simply the composite of the inverse of the glide reflection mentioned and the second translation.)

The actual elements that generate the group are obtained by the method discussed at the beginning of $\S 3$. A graph is convenient for this purpose: each vertex represents a translate of $Q$ and the label on the vertex indicates by which element $Q$ was moved. for example, the horizontal edges in the top graph show that if we pass through $S_{++00}$, we arrive in $a^{-1} Q$; passing further through $a^{-1} S_{--00}$ gets us in $a^{-1} b Q$.

Since the group of rotational parts of $G_{v_{+000}}$ has order 2, the subgroup of translations has index 2 in $G_{v_{+000}}$, which means that $Q_{v_{+000}} \cup\left(a^{-1} g\right) Q_{v_{+000}}$ is a fundamental polyhedron for this subgroup. It is visually clear that this polyhedron will fill out $\mathbf{R}^{3}$ if it is moved around by composites of translations $t_{1}=c, t_{2}=a^{-1} b$ and $t_{3}=h^{-1} g$, hence those three translations generate the subgroup of translations $\left(h^{-1} g\right.$ is a translation using the same explanation as $\left.a^{-1} b\right)$. The normal translations among them are those whose vectors are \pm 1 eigenvectors of 

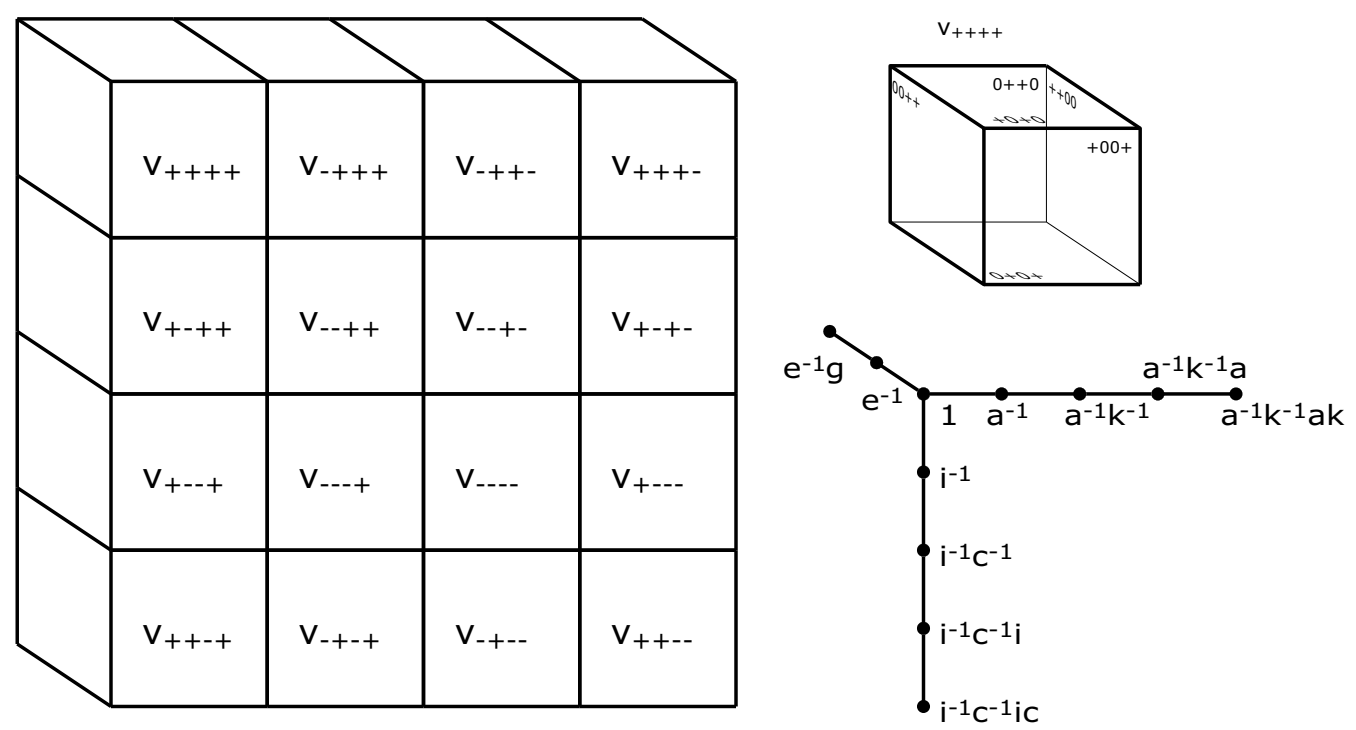

Figure 3: Fundamental polyhedron for $G_{v_{+++}}$and generators of its translation subgroup

the reflection in $x_{3}=0$, which are multiples of $t_{1}$ and any combination of $t_{2}$ and $t_{3}$.

Fig. 3 shows the fundamental polyhedron $Q_{v_{++++}}$. The smaller cube illustrates how the faces are labeled on the portion of $Q_{v_{++++}}$labeled $v_{++++}$; this labeling implies the labeling on every other cube that makes up $Q_{v_{+++}+}$. For example, the front side on cube $v_{----}$is labeled -00-, we simply insert zeroes in the appropriate places into the string labeling $v_{* * * *}$. Using techniques as above (or see $§ 5$ ) it is easy to see that the left and right sides of the rectangular box, as well as the bottom and top sides are paired by translations. The side pairing pattern for the back side is illustrated in Fig. 4 and the pattern for the front side is identical. Note that shaded rectangles are paired by a glide reflection. To see this, note that $S_{0++0}$ is paired to $S_{0--0}$ via $e$. Since $e v_{++++}=v_{----}$the back side of the cube labeled $v_{++++}$is paired to the back side of the cube $v_{----}$. As in 4.1 , take a path from the cube labeled $v_{++++}$that goes two steps to the right, then two steps down and then exits out the back of the cube labeled $v_{----}$. The composite of the reflections in the first four planes that we pass through gives a translation in the direction of arrow in Fig. 4, composing with a reflection in the back plane makes it a glide translation. We also have to compose this with a conjugate of a certain element of $K$ which turns out to be 1 because the only element of $K$ that sends $v_{++++}$to itself is 1 . The translation subgroup of $G_{v_{+++}}$is obtained in the same way as for $G_{v_{+000}}$ above.

We obtain all the relations and all the generators of the translation subgroups for each 


\begin{tabular}{|c|c|c|c|}
\hline$A$ & $B$ & $E$ & $F$ \\
\hline$C$ & $D$ & $G$ & $H$ \\
\hline$E^{\prime}$ & $F^{\prime}$ & $A^{\prime}$ & $B^{\prime}$ \\
\hline$G^{\prime}$ & $H^{\prime}$ & $C^{\prime}$ & $D^{\prime}$ \\
\hline
\end{tabular}

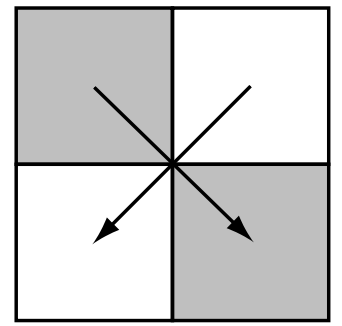

glide reflections

(reflection is in the

plane containing

the sides)

Figure 4: Side-pairing pattern of front and back sides of $Q_{v_{++++}}$

vertex cycle in the way described above. Here are the relations:

$$
\left.\left.\begin{array}{rl}
b^{-1} d^{-1} b c & =1 \\
a^{-1} d^{-1} a c & =1 \\
b^{-1} h a^{-1} g & =1 \\
b^{-1} g a^{-1} h & =1 \\
g^{-1} d g c & =1 \\
h^{-1} d h c & =1
\end{array}\right\} \text { from } Q_{v_{+000}} \begin{array}{rl}
j^{-1} b^{-1} j a & =1 \\
i^{-1} b^{-1} i a & =1 \\
j^{-1} f i^{-1} e & =1 \\
j^{-1} e i^{-1} f & =1 \\
e^{-1} b e a & =1 \\
f^{-1} b f a & =1
\end{array}\right\} \text { from } Q_{v_{0+00}}
$$

Recall that every edge of $Q$ has an ideal vertex that is among $v_{* 000}, v_{0 * 00}, v_{00 * 0}$ and $v_{000 *}$, hence every cycle of edges of $Q$ is represented by a cycle of edges of one of $Q_{v_{+000}}, Q_{v_{0+00}}$, $Q_{v_{00+0}}$ and $Q_{v_{000+}}$. Cycles of edges of $Q_{v_{+++}}$will not add any new relations, so they can be omitted. 
Now we list all the generating translations for each parabolic subgroup.

subgroup generating translations normal translations

\begin{tabular}{|c|c|c|}
\hline$G_{v_{+000}}$ & $\begin{array}{l}t_{1}=c \\
t_{2}=a^{-1} b \\
t_{3}=h^{-1} g\end{array}$ & $\begin{array}{l}n t_{1} \\
m t_{2}+n t_{3}\end{array}$ \\
\hline$G_{v_{0+00}}$ & $\begin{array}{l}t_{1}=a \\
t_{2}=i^{-1} j \\
t_{3}=f^{-1} e\end{array}$ & $\begin{array}{l}n t_{1} \\
m t_{2}+n t_{3}\end{array}$ \\
\hline$G_{v_{00+0}}$ & $\begin{array}{l}t_{1}=k \\
t_{2}=c^{-1} d \\
t_{3}=f e\end{array}$ & $\begin{array}{l}n t_{1} \\
m t_{2}+n t_{3}\end{array}$ \\
\hline$G_{v_{000+}}$ & $\begin{array}{l}t_{1}=i \\
t_{2}=k^{-1} l \\
t_{3}=h g\end{array}$ & $\begin{array}{l}n t_{1} \\
m t_{2}+n t_{3}\end{array}$ \\
\hline$G_{v_{++++}}$ & $\begin{aligned} t_{1} & =e^{-1} g \\
t_{2} & =a^{-1} k^{-1} a k \\
t_{3} & =i^{-1} c^{-1} i c\end{aligned}$ & $\begin{array}{l}n t_{1} \\
m t_{2}+n t_{3}\end{array}$ \\
\hline
\end{tabular}

We now choose translations in each boundary component that we want to represent the fiber of the $S^{1}$-bundle. We simply go for the easiest expression in order to simplify computation. Choose $c^{n}, a, k, i$ and $e^{-1} g$ and compute $\pi_{1} M /\left\langle\left\langle c^{n}, a, k, i, e^{-1} g\right\rangle\right\rangle$. Substituting $a, k, i=1$ into the four groups of relators we get the following four respective groups:

$$
\left.\left.\begin{array}{rl}
b^{-1} d^{-1} b c & =1 \\
d & =c \\
b^{-1} h g & =1 \\
b^{-1} g h & =1 \\
g^{-1} d g c & =1 \\
h^{-1} d h c & =1
\end{array}\right\} \begin{array}{rl}
b & =1 \\
j^{-1} f e & =1 \\
j^{-1} e f & =1
\end{array}\right\}
$$


Taking into account now that $l, b, j, e^{-1} g=1$ and $c=d$, we get the relations:

$$
h=g^{-1}, h=g, g=e, f=e^{-1}, g^{-1} c g c=1, c^{-1} e c^{-1} e=1
$$

which immediately implies that the group is generated by $c$ and $e$, and that $e^{2}=1, e^{-1} c e c=1$ and $c^{-1} e c^{-1} e=1$. The last two equations are both equivalent to $e^{-1} c e=c^{-1}$ so the presentation of the group is $\left\langle c, e \mid c^{n}=1, e^{2}=1, e^{-1} c e=c^{-1}\right\rangle=\left\langle c \mid c^{n}=1\right\rangle \odot\left\langle e \mid e^{2}=1\right\rangle=$ $\mathbf{Z}_{n} \odot \mathbf{Z}_{2}$, where $\mathbf{Z}_{2}$ acts on $\mathbf{Z}_{n}$ by inversion in the semidirect product $\mathbf{Z}_{n} \odot \mathbf{Z}_{2}$. Letting $H=\left\langle\left\langle c^{n}, a, k, i, e^{-1} g\right\rangle\right\rangle$, we note that $c^{n}$ is primitive in $H \cap G_{v_{+000}}, a$ is primitive in $H \cap G_{v_{0+00}}$ and so on. Proposition 2.4 now tells us that $M$ has a $2 n$-fold cover $\tilde{M}$ that is a complement in a simply-connected manifold $N$.

When $n=1$ the two-fold cover of $M$ is actually the orientable double cover of $M$, since via the homomorphism $\pi_{1} M \rightarrow \pi_{1} M /\left\langle\left\langle c, a, k, i, g^{-1} e\right\rangle\right\rangle \cong \mathbf{Z}_{2}$ the orientation reversing generators $e, f, g, h$ of $G$ are sent to the nonidentity element of $\mathbf{Z}_{2}$ while the orientation preserving generators $a, b, c, d, i, j, k, l$ are sent to the identity element. Furthermore, $\chi(N)=$ $\chi($ double cover of $M)=2$. Since $H_{4} N=H_{0} N=\mathbf{Z}$ and $H_{1} N=H_{3} N=0$ this means $\mathrm{H}_{2} \mathrm{~N}=0$. Now due to Freedman's (see $[2,3]$ ) classification of simply-connected 4-manifolds, the only simply-connected 4 manifold $\mathrm{N}$ with $\mathrm{H}_{2} \mathrm{~N}=0$ is the 4-sphere.

When $n>2$, let $\pi_{1} M \stackrel{q}{\rightarrow} \mathbf{Z}_{n} \odot \mathbf{Z}_{2}$ be the quotient homomorphism. The orientationreversing generators of $\pi_{1} M$ are sent to the generator of $\mathbf{Z}_{2}$, so the subgroup of orientationpreserving transformations of $\pi_{1} M$ is $q^{-1}\left(\mathbf{Z}_{n}\right)$. Since $\operatorname{ker} q \subset q^{-1}\left(\mathbf{Z}_{n}\right)$, the $2 n$-fold cover corresponding to ker $q$ covers the orientable double cover which corresponds to $q^{-1}\left(\mathbf{Z}_{n}\right)$. The group of deck transformations is $q^{-1}\left(\mathbf{Z}_{n}\right) / \operatorname{ker} q \cong \mathbf{Z}_{n}$ so $\tilde{M}$ is a cyclic cover of the double orientable cover.

We use Proposition 2.3 to find the number of components of $\tilde{M}$. The subgroup $G_{v_{+000}}$ is generated by translations $c$ and $a^{-1} b$ and an orientation-reversing glide reflection. Those transformations are respectively sent to $c, 1, e \in \mathbf{Z}_{n} \odot \mathbf{Z}_{2}$, hence the index of $q\left(G_{v_{+000}}\right)$ in $\mathbf{Z}_{n} \odot \mathbf{Z}_{2}$ is 1 . Similarly, we see that $q\left(G_{v_{0+00}}\right)=q\left(G_{v_{00+0}}\right)=q\left(G_{v_{000+}}\right)=q\left(G_{v_{++++}}\right)=\langle e\rangle$ so the index of each of those subgroups of $\mathbf{Z}_{n} \odot \mathbf{Z}_{2}$ is $n$. Therefore, $\tilde{M}$ has $4 n+1$ boundary components.

Thus we have proved

Theorem 4.3 Let $M$ be the Ratcliffe-Tschantz manifold no. 1011. Then $M$ is a complement of 5 tori in a 4-manifold $N$ with $\pi_{1} N=\mathbf{Z}_{2}$. The orientable double cover of $M$ is the complement of 5 tori in $S^{4}$. Furthermore, the orientable double cover has cyclic covers of any index $n$ that are complements of $4 n+1$ tori in a simply-connected closed manifold $N$ with $\chi(N)=2 n$. 


\section{A necessary condition}

With a wealth of Ratcliffe-Tschantz examples available, one would like to know whether many have a double cover that is a complement in $S^{4}$ or if this is a rare occurence. We give a criterion that rules out an example from having such a double cover that (unfortunately) applies to most Ratcliffe-Tschantz examples.

Proposition 5.1 Let $M$ be a hyperbolic manifold that has a double cover which is a complement in a simply-connected closed manifold. Then there exist translations $t_{1}, \ldots, t_{m}$ so that $\pi_{1} M /\left\langle\left\langle t_{1}, \ldots, t_{m}\right\rangle\right\rangle=\mathbf{Z}_{2}$.

Proof. Let $H \subset \pi_{1} M$ be the subgroup of $\pi_{1} M$ corresponding to the double cover in question. Since the double cover is a complement there exist translations normal in $H$ so that $H /\left\langle\left\langle t_{1}, \ldots, t_{m}\right\rangle\right\rangle_{H}=1$ (see proposition 2.2). However, $H$ is normal in $\pi_{1} M$ (it has index 2) so $H=\left\langle\left\langle t_{1} \ldots t_{m}\right\rangle\right\rangle_{H} \subset\left\langle\left\langle t_{1} \ldots t_{m}\right\rangle\right\rangle \subset H$ which immediately gives $\left\langle\left\langle t_{1} \ldots t_{m}\right\rangle\right\rangle=H$ and the conclusion of the proposition.

Note that the double cover of $M$ may have more boundary components than $M$, hence, in general, $m$ is greater of equal to the number of boundary components of $M$.

Now we consider a Ratcliffe-Tschantz manifold $M$ and use the notation of $\S 4$. Let $G=$ $\pi_{1} M$ and let $\mathbf{Z}_{2}^{6}$ be the abelian group whose summands are generated by the 6 order-2 elements $r_{* * 00}, r_{* 0 * 0}, r_{0 * * 0}, r_{* 00 *}, r_{0 * 0 *}, r_{00 * *}$. Note that only here "*" is a symbol and not a slot allowing other symbols. As we have mentioned in $\S 4, G \subset \Gamma_{2}^{4}$, where $\Gamma_{2}^{4}$ is generated by reflections in the sides of the polyhedron $P$ whose angles are all $\pi / 2$. There is a homomorphism $\phi: \Gamma_{2}^{4} \rightarrow \mathbf{Z}_{2}^{6}$ given by $k_{* * * *} \mapsto 0, r_{++00} \mapsto r_{* * 00}, r_{+0+0} \mapsto r_{* 0 * 0}$, etc. We restrict $\phi$ to $G$ and note that $\phi: G \rightarrow \mathbf{Z}_{2}^{6}$ is surjective: the side-pairing for side $S_{++00}$ maps to $r_{* * 00}$, the side-pairing for $S_{+0+0}$ maps to $r_{* 0 * 0}$ and so on.

Referring to the 24 -sided polyhedron $Q$ from $\S 4$, let $v$ be any of the ideal vertices that has only one positive nonzero position. If $C$ is a horosphere centered at $v$, then $C \cap Q$ is a cube and translates of $Q$ that intersect give a tiling of $C \cong \mathbf{R}^{3}$ by cubes. Note that the parallel sides of the cube $Q \cap C$ are sides of the polyhedron $Q$ that have nonzero entries in the same position, so their side-pairings have the same $k$ in the $k r$ decomposition of proposition 3.1.

In what follows, like in proposition 4.1 , we drop " $\cap C$ ". If we start a path in the cube $Q$ that exits through side $S$, goes through the adjacent cube $x Q$ and then exits through the parallel side opposite the entry side, finishing in translate $g Q$, the transformation $g$ is equal to $q_{2} q_{1} k_{1}^{-1} k_{2}^{-1}$ according to proposition 4.1. However, since side-pairings for parallel sides have the same $K$-part, $k_{1}=k_{2}$, so we get $g=q_{2} q_{1}$, where $q_{1}$ and $q_{2}$ are reflections in planes that we passed through. It then follows that $g$ is a translation. Also, $\phi(g)=0$ 
since both $q_{1}$ and $q_{2}$ map to the same generator of $\mathbf{Z}_{2}^{6}$ (every reflection $q_{i}$ is a conjugate of $r_{++00}, r_{0+0+}, \ldots, r_{00++}$ by elements of $\left.K\right)$.

Therefore, translations by two lengths of the side of $Q$ in each of the three directions parallel to the edges of $Q$ are contained in the translation subgroup of $G_{v}$. Let $T_{v}$ denote the group of translations they generate. Since the fundamental polyhedron for $G_{v}$ is two sideby-side cubes and the fundamental polyhedron for $T_{v}$ is eight cubes, we get $\left[G_{v}: T_{v}\right]=4$. Note also that $\phi\left(T_{v}\right)=0$.

Similarly, if $v$ is the ideal vertex $v_{++++}$and $C$ a horosphere centered at $v$, then $Q \cap C$ is again a cube whose parallel sides have nonzero entries in complementary positions. A path that goes perpendicularly through a side of the cube and continues in a straight line until it has crossed 4 sides finishes in the translate $g Q$, where (proposition 4.1 again) $g=$ $q_{4} q_{3} q_{2} q_{1} k_{1}^{-1} k_{2}^{-1} k_{3}^{-1} k_{4}^{-1}$. However, since the $K$-part of a side-pairing is the same for every side that has nonzero entries in fixed positions, we have $k_{1}=k_{3}$ and $k_{2}=k_{4}$, so $g=q_{4} q_{3} q_{2} q_{1}$ owing to commutativity of $K=\mathbf{Z}_{2}^{4}$ and the fact that every element of $K$ has order 2. This makes $g$ a translation by four lengths of a side of $Q$ as it is the composite of four reflections in parallel planes. Also, $\phi(g)=0$ because $\Phi\left(k_{i}\right)=0, \Phi\left(q_{1}\right)=\Phi\left(q_{3}\right)$ and $\Phi\left(q_{2}\right)=\Phi\left(q_{4}\right)$. Again let $T_{v}$ be the subgroup of translations generated by translations by four cube widths in the three directions parallel to the edges of the cube $Q$. The fundamental polyhedron of $T_{v}$ comprises 64 cubes, while the fundamental polyhedron of $G_{v}$ contains either 16 cubes, if $|[v]|=16$, or 8 cubes if $|[v]|=8$. Thus, $\left[G_{v}: T_{v}\right]=4$ or 8 , respectively. Again note that $\phi\left(T_{v}\right)=0$.

Theorem 5.2 Let $M$ be a Ratcliffe-Tschantz manifold and let $T$ denote the set of all parabolic translations in $G=\pi_{1} M$. Viewing $\mathbf{Z}_{2}^{6}$ as a $\mathbf{Z}_{2}$-vector space, if the dimension of the subspace generated by $\phi(T)$ is less than 5 , then $M$ does not have a double cover that is a complement in $S^{4}$.

In particular, let $M$ be a 5-cusped Ratcliffe-Tschantz manifold, let $n_{X}$ denote the number of boundary components (corresponding to cusps) of $M$ of type $X, X=A, B, F, G, H, I, J$ and let $\alpha(M)=2 n_{A}+2 n_{B}+n_{G}+n_{H}$. If $\alpha(M)<5$ then $M$ does not have a double cover that is a complement in $S^{4}$.

For a 6-cusped Ratcliffe-Tschantz manifold $M$, let $n_{X}^{\prime}$ denote the number of boundary components of type $X$ that correspond to the two cycles of the ideal vertices $v_{ \pm, \pm, \pm, \pm}$(the last two letters in Ratcliffe and Tschantz's table of 6-cusped manifolds are the ones that identify boundary component types corresponding to those vertices) and let $n_{X}$ denote the number of boundary components of type $X$ that correspond to the 4 cycles of the remaining ideal vertices. Set $\alpha^{\prime}(M)=2 n_{A}+2 n_{B}+n_{G}+n_{H}+2 n_{A}^{\prime}+2 n_{B}^{\prime}+n_{F}^{\prime}+n_{G}^{\prime}+n_{H}^{\prime}+n_{I}^{\prime}+n_{J}^{\prime}$. If $\alpha^{\prime}(M)<5$ then $M$ does not have a double cover that is a complement in $S^{4}$. 
Proof. We prove the contrapositive. Suppose $M$ has a double cover that is a complement in a simply-connected manifold and let $G=\pi_{1} M$. According to Proposition 5.1 there exist translations $t_{1}, \ldots, t_{m}$ so that $\pi_{1} M /\left\langle\left\langle t_{1}, \ldots, t_{m}\right\rangle\right\rangle=\mathbf{Z}_{2}$. The homorphism $\phi: G \rightarrow \mathbf{Z}_{2}^{6}$ defined above induces a surjective homomorphism $G /\left\langle\left\langle t_{1} \ldots t_{m}\right\rangle\right\rangle \rightarrow \mathbf{Z}_{2}^{6} /\left\langle\phi\left(t_{1}\right) \ldots \phi\left(t_{m}\right)\right\rangle \rightarrow$ $\mathbf{Z}_{2}^{6} /\langle\phi(T)\rangle$ so $\operatorname{dim} \mathbf{Z}_{2}^{6} /\langle\phi(T)\rangle \leq 1$ which gives $\operatorname{dim}\langle\phi(T)\rangle \geq 5$.

For the remaining two parts, we notice first that if the type of the boundary component is $F, I$ or $J$ and $G_{v}$ is its corresponding parabolic subgroup, then the index of the translation subgroup of $G_{v}$ is 4 . Hence, if the index of $T_{v}$ in $G_{v}$ is 4 , then the translation subgroup of $G_{v}$ is equal to $T_{v}$.

Now suppose that $M$ is a 5 -cusped Ratcliffe-Tschantz manifold that has a double cover $\tilde{M}$ that is a complement in $S^{4}$. By Proposition 5.1 there exist $t_{1}, \ldots, t_{m}$ so that $G /\left\langle\left\langle t_{1}, \ldots, t_{m}\right\rangle\right\rangle=$ $\mathbf{Z}_{2}$. Any translation $t_{i}$ that is in a parabolic subgroup corresponding to a boundary component of type $F, I$ or $J$ maps to 0 via $\phi$ by the above discussion, since the index of $T_{v}$ in $G_{v}$ is always 4 in the 5 -cusped case. Boundary components of type $G$ and $H$ are unorientable, so their lifts in $\tilde{M}$ are orientable and thus have only one component; this means that only one translation in the collection $t_{1}, \ldots, t_{m}$ is in the parabolic subgroup corresponding to a boundary component of type $G$ or $H$. Boundary components of type $A$ or $B$ could have two components in their lifts and each component could have a different choice for fiber of the $S^{1}$-fiber bundle, therefore, at most two translations in the collection $t_{1}, \ldots, t_{m}$ are in the parabolic subgroup corresponding to each boundary component of type $A$ or $B$. Therefore, the number of nonzero elements of $\phi\left(t_{1}\right), \ldots, \phi\left(t_{m}\right)$ is at most $2 n_{A}+2 n_{B}+n_{G}+n_{H}$. Again, surjectivity of the induced map $G /\left\langle\left\langle t_{1} \ldots t_{m}\right\rangle\right\rangle \rightarrow \mathbf{Z}_{2}^{6} /\left\langle\phi\left(t_{1}\right) \ldots \phi\left(t_{m}\right)\right\rangle$ gives us that $2 n_{A}+2 n_{B}+n_{G}+n_{H} \geq \operatorname{dim}\left\langle\phi\left(t_{1}\right), \ldots, \phi\left(t_{m}\right)\right\rangle \geq 5$ proving the contrapositive of the second claim.

If $M$ is a 6-cusped Ratcliffe-Tschantz manifold, we reason in the same fashion. For a vertex in a cycle of $v_{ \pm, \pm, \pm \pm}$the index of $T_{v}$ in $G_{v}$ is 8 , hence $T_{v}$ has index 2 in the translation subgroup of $G_{v}$. Therefore, even if the boundary component type is $F, I$ or $J$, there could be a nonzero element corresponding to it in the collection $\phi\left(t_{1}\right), \ldots, \phi\left(t_{m}\right)$ and, as above, there could be two if the boundary component is orientable. Thus, the number of nonzero elements in $\phi\left(t_{1}\right), \ldots, \phi\left(t_{m}\right)$ is at most $2 n_{A}+2 n_{B}+n_{G}+n_{H}+2 n_{A}^{\prime}+2 n_{B}^{\prime}+n_{F}^{\prime}+n_{G}^{\prime}+n_{H}^{\prime}+n_{I}^{\prime}+n_{J}^{\prime}$ and the proof is finished as in the 5 -cusped case. (Note that, since the boundary component types corresponding to the two cycles of $v_{ \pm, \pm, \pm, \pm}$are always equal, only one of the numbers $n_{A}^{\prime}, n_{B}^{\prime}, n_{F}^{\prime}, n_{G}^{\prime}, n_{H}^{\prime}, n_{I}^{\prime}, n_{J}^{\prime}$ is nonzero.) 
Example 5.3 Proposition 5.2 rules out easily many Ratcliffe-Tschantz examples to get that at most about 346 (of 1171) can have double cover complements in $S^{4}$.

First of all, any nonorientable example that contains a boundary component of type $F$ cannot have a double cover complement in $S^{4}$, since the boundary component will lift to two copies of boundary components of type $F$ which are not $S^{1}$-bundles. A hand count that rules out all nonorientable examples with a boundary component of type $F$ and rules out all manifolds that satisfy the second or the third part of Proposition 5.2 leaves 18 (of 22) 5-cusped orientable manifolds, about 287 (of 1068) 5-cusped nonorientable and 41 (of 81) 6-cusped nonorientable manifolds eligible to be complements inside $S^{4}$. For example, if $M_{k}$ denotes the Ratcliffe-Tschantz manifold(s) ranging through $k$ 's, we have $\alpha\left(M_{19-22}\right)=4$, $\alpha\left(M_{811-865}\right) \leq 2, \alpha^{\prime}\left(M_{1101-1104}\right)=4$.

Example 5.4 There are many Ratcliffe-Tschantz manifolds that can be ruled out using the first, stronger, part of Proposition 5.2 by computing $\operatorname{dim} \phi(T)$. For example, while $\alpha\left(M_{51}\right)=7$, it is not hard to compute that $\operatorname{dim} \phi(T)=4$. Similarly, $\alpha\left(M_{545}\right)=6$, and $\alpha^{\prime}\left(M_{1162}\right)=10$, yet $\operatorname{dim} \phi(T)$ is 3 and 4 , respectively.

We have used the first part Proposition 5.2 to investigate many potential candidates for complements in $S^{4}$ and have been able to rule most of them out. Some examples remain that cannot be excluded in this way and they are possible candidates for complements. However, we feel that only a few Ratcliffe-Tschantz examples will turn out to have double cover complements in $S^{4}$ and have confirmed only the one from Theorem 4.3.

\section{Remark}

In the proof of Theorem 4.3 we used Freedman's theory which applies to the topological category, that is, we can only claim that the double cover of $M$ from Theorem 4.3 embeds in a space $N$ that is homeomorphic to $S^{4}$. At this time it is still unknown whether a manifold that is homeomorphic to $S^{4}$ is also diffeomorphic to the $S^{4}$ with the standard differentiable structure (the "differentiable Poincaré conjecture in dimension 4"). If we had a handle decomposition for $N$, diffeomorphism with $S^{4}$ can be verified by using Kirby calculus to transform the Kirby diagram of $N$ to the Kirby diagram of the standard $S^{4}$. It is not too hard to produce a Kirby diagram of the manifold if it is given by a side-pairing of a polyhedron (spherical, euclidean or hyperbolic). Then one can obtain a Kirby diagram for $N$, which turns out to be rather complicated (it has 23 1-handles, 53 2-handles, 34 3-handles and 54 -handles). A computation using Kirby moves shows that $N$ really is diffeomorphic to $S^{4}$ — we plan to write the details in a future paper.

Hence, we have produced a complicated Kirby diagram of $S^{4}$ obtained in a seemingly nontrivial, top-down fashion (as opposed to a bottom-up method where one would start with 
a simple Kirby diagram of the standard $S^{4}$ and make it more complicated via Kirby moves). While of course this does not give a counterexample to the differentiable Poincaré conjecture in dimension 4 , it seems to indicate that a possible counterexample given by a Kirby diagram may indeed be very complicated. 


\section{References}

[1] B.N. Apanasov. Conformal geometry of Discrete Groups and Manifolds. de Gruyter Expositions in Mathematics. Walter de Gruyter, 2000.

[2] M. H. Freedman and F. Quinn. Topology of 4-Manifolds. Princeton University Press, 1990.

[3] R. Gompf and A. Stipsicz. 4-manifolds and Kirby Calculus, volume 20 of Graduate Studies in Mathematics. AMS.

[4] D. Ivanšić. Finite-volume hyperbolic 4-manifolds that share a fundamental polyhedron. Diff. Geom. and Appl., 10(3):205-223, 1999.

[5] D. Ivanšić. Embeddability of noncompact hyperbolic manifolds as complements of codimension-1 and -2 submanifolds. Topology Appl., 120(1-2):211-236, 2002. In memory of T. Benny Rushing.

[6] B. Nimershiem. All flat three-manifolds appear as cusps of hyperbolic four-manifolds. Topology Appl., 90(1-3):109-133, 1998.

[7] J. Ratcliffe. Foundations of hyperbolic manifolds. Springer-Verlag, 1994.

[8] J. Ratcliffe and S. Tschantz. The volume spectrum of hyperbolic 4-manifolds. Experiment. Math., 9(1):101-125, 2000.

[9] W. Thurston. The geometry and topology of three-manifolds. Princeton University, 1979. lecture notes.

[10] H. C. Wang. Topics on totally discontinuous groups, volume Pure Appl. Math. Vol. 8, pages 460-485. Marcel-Dekker, 1972.

[11] N. Wielenberg. The structure of certain subgroups of the picard group. Math. Proc. Cambridge Phil. Soc., 84:427-436, 1978.

Dubravko Ivanšić

Department of Mathematics and Statistics

Murray State University

Murray, KY 42071

dubravko.ivansic@murraystate.edu 